\title{
Correction to: Differences in cancer survival by sex: a population- based study using cancer registry data
}

\author{
Nina Afshar ${ }^{1,2} \oplus \cdot$ Dallas R. English $^{1,2} \cdot$ Vicky Thursfield $^{3} \cdot$ Paul L. Mitchell $^{4} \cdot$ Luc Te Marvelde $^{1,5} \cdot$ Helen Farrugia $^{3} \cdot$ \\ Graham G. Giles ${ }^{1,2} \cdot$ Roger L. Milne ${ }^{1,2}$
}

Published online: 11 October 2018

(c) Springer Nature Switzerland AG 2018

\section{Correction to: Cancer Causes \& Control https://doi.org/10.1007/s10552-018-1079-z}

In the original publication of the article, the concluding paragraph of the Discussion section was inadvertently missed and is provided below.

In conclusion, the current study showed that in Australia, men generally fare worse with cancer than women. It is essential to unravel the mediating effects of stage at diagnosis, co-morbidities, treatment modalities and health-related lifestyle factors on these sex differences in cancer outcomes. Emerging new methods from the causal inference literature offer promise in this area, particularly as access to linked population-based cancer and other health-related datasets improves. Future national and international research should focus on identifying actionable factors to address inequalities in cancer outcomes, which may improve survival for both men and women.

The original article can be found online at https://doi.org/10.1007/ s10552-018-1079-z.

Nina Afshar

Nina.Afshar@cancervic.org.au

1 Cancer Epidemiology and Intelligence Division, Cancer Council Victoria, 615 St Kilda Road, Melbourne, VIC 3004, Australia

2 Centre for Epidemiology and Biostatistics, School of Population and Global Health, The University of Melbourne, 207 Bouverie Street, Melbourne, VIC 3010, Australia

3 Victorian Cancer Registry, Cancer Council Victoria, $615 \mathrm{St}$ Kilda Road, Melbourne, VIC 3004, Australia

4 Department of Medical Oncology, Austin Health, Olivia Newton-John Cancer Wellness and Research Centre, 145 Studley Road, Heidelberg, VIC 3084, Australia

5 Cancer Strategy and Development, Department of Health and Human Services, 50 Lonsdale Street, Melbourne, VIC 3000, Australia 